\title{
Surgical outcomes of modified lumbar spinous process-splitting laminectomy for lumbar spinal stenosis
}

\author{
Shunsuke Kanbara, MD, ${ }^{1}$ Yasutsugu Yukawa, MD, ${ }^{2}$ Keigo Ito, MD, ${ }^{2}$ Masaaki Machino, MD, ${ }^{2}$ \\ and Fumihiko Kato, MD²
}

\begin{abstract}
1Department of Orthopedic Surgery, Chutoen General Medical Center, Shizuoka; and 2Department of Orthopedic Surgery, Chubu Rosai Hospital, Nagoya, Japan
\end{abstract}

The lumbar spinous process-splitting laminectomy (LSPSL) procedure was developed as an alternative to lumbar laminectomy. In the LSPSL procedure, the spinous process is evenly split longitudinally and then divided at its base from the posterior arch, leaving the bilateral paravertebral muscle attached to the lateral aspects. This procedure allows for better exposure of intraspinal nerve tissues, comparable to that achieved by conventional laminectomy while minimizing damage to posterior supporting structures. In this study, the authors make some modifications to the original LSPSL procedure (modified LSPSL), in which laminoplasty is performed instead of laminectomy. The purpose of this study was to compare postoperative outcomes in modified LSPSL with those in conventional laminectomy $(\mathrm{CL})$ and to evaluate bone unions between the split spinous process and residual laminae following modified LSPSL.

Forty-seven patients with lumbar spinal stenosis were enrolled in this study. Twenty-six patients underwent modified LSPSL and 21 patients underwent CL. Intraoperative blood loss and surgical duration were evaluated. The Japanese Orthopaedic Association (JOA) scale scores were used to assess parameters before surgery and 12 months after surgery. The recovery rates were also evaluated. Postoperative paravertebral muscle atrophy was assessed using MRI. Bone union rates between the split spinous process and residual laminae were also examined.

The mean surgical time and intraoperative blood loss were 25.7 minutes and $42.4 \mathrm{ml}$ per 1 level in modified LSPSL, respectively, and 22.7 minutes and $29.5 \mathrm{ml}$ in CL, respectively. The recovery rate of the JOA score was $64.2 \%$ in modified LSPSL and $68.7 \%$ in CL. The degree of paravertebral muscle atrophy was $7.8 \%$ in modified LSPSL and $22.2 \%$ in CL at 12 months after surgery $(p<0.05)$. The fusion rates of the spinous process with the arcus vertebrae at 6 and 12 months in modified LSPSL were $56.3 \%$ and $81.3 \%$, respectively.

The modified LSPSL procedure was less invasive to the paravertebral muscles and could be a laminoplasty; therefore, the modified LSPSL procedure presents an effective alternative to lumbar laminectomy.

http://thejns.org/doi/abs/10.3171/2014.9.SPINE1457

KEY WORDS lumbar spinous process-splitting laminectomy; laminoplasty; lumbar spinal stenosis; decompression surgery; spinous process; paravertebral muscle

$\mathrm{C}$ URRENTLY, there are several surgical approaches to correct lumbar spinal stenosis (LSS). Although conventional laminectomy for LSS has been routinely performed, this procedure involves the dissection of the bilateral paravertebral muscles (PVMs) that are detached extensively from the spinous process and laminae. In addition, the posterior midline ligaments, such as the supraand interspinous ligaments, lose their original attachments when the spinous processes are removed. Surgical damage to these important stabilizing structures may lead to post- operative segmental malalignment, instability, and subsequently failed-back surgery syndrome. To prevent such damaging complications, Watanabe et al ${ }^{17}$ first developed lumbar spinous process-splitting laminectomy (LSPSL). This procedure involves longitudinally splitting the spinous process into two halves, leaving its muscular and ligamentous attachments undisturbed, followed by laminectomy with minimal muscle dissection from the lamina. The LSPSL procedure allows for sufficient exposure of intraspinal nerve tissues, comparable with conventional

ABBREVIATIONS CL = conventional laminectomy; JOA = Japanese Orthopaedic Association; LSPSL = lumbar spinous process-splitting laminectomy; LSS = lumbar spinal stenosis; $\mathrm{PVM}=$ paravertebral muscle.

SUBMITTED January 22, 2014. ACCEPTED September 19, 2014

INCLUDE WHEN CITING Published online January 16, 2015; DOI: 10.3171/2014.9.SPINE1457.

DISCLOSURE The authors report no conflict of interest concerning the materials or methods used in this study or the findings specified in this paper. 
laminectomy (CL), while minimizing damage to posterior supporting structures ${ }^{17}{ }^{17}$ However, the split spinous process loses the residual laminae; we modified this procedure, retaining continuity between the split spinous process and laminae. The purpose of this study was to compare surgical outcomes in modified LSPSL with those of CL and to evaluate the usefulness of the modified LSPSL procedure.

\section{Methods}

Patient inclusion criteria were lumbar canal stenosis, no degenerative lumbar spondylolisthesis, and no previous history of lumbar surgery. Patients with degenerative spondylolisthesis and degenerative lumbar scoliosis were excluded from this study. From 2005 to 2008, 47 eligible patients underwent stenosis decompression. All patients were followed-up for more than 1 year. We evaluated the outcomes of 26 patients (18 men and 8 women, mean age 68.3 years, range $53-84$ years) who underwent modified LSPSL from May 2007 to October 2008 and 21 patients (11 men and 10 women, mean age 69.4 years, range 47-82 years) who underwent CL from January 2005 to May 2007 (Table 1). In the modified LSPSL procedure, decompression was performed at 2 levels in 13 patients, 3 levels in 8 patients, and 4 levels in 5 patients. In the CL procedure, decompression was performed at 2 levels in 10 patients, 3 levels in 9 patients, and 4 levels in 2 patients. In all patients, the surgery was performed by the experienced senior author (Y.Y.). This study was approved by the Institutional Review Board of Chubu Rosai Hospital.

\section{Modified Surgical Procedures}

A total of 26 patients underwent modified LSPSL in the prone position. In this procedure, a posterior midline skin incision was made between the L-3 and L-5 spinous processes to expose the top of the L- 4 spinous process (if L3-4 and L4-5 decompressions were predetermined). The midline of the supraspinous and interspinous ligaments of both L3-4 and L4-5 were cut; the L-4 spinous process was longitudinally split to the middle of its base with an electric bone saw and then bilaterally divided from the L-4 laminae at the planned angle with a flat chisel to slip off the upper half of the lamina, thereby leaving the bilateral PVM attached to the lateral aspect of the split spinous process (Fig. 1A). The supra- and interspinous ligaments between L3-4 and L4-5 were also split to the termini. A sufficiently wide working space for an L-4 laminectomy was obtained by retracting the split spinous process bilaterally with its PVM (Fig. 1B). The decompression procedure was the same as the procedure of Watanabe et al. ${ }^{17}$ The L-4 laminae and the small lower part of the L-3 laminae were removed using both flat and circular chisels. The L3-4 ligamentum flavum was then excised from the L3-4 interspace. The ligamentum flavum beneath the posterior aspect of the L-3 laminae was removed using a Kerrison rongeur. If good access to the lateral recesses and the entry zone of the intervertebral foramina was obtained, the nerve roots and thecal sac were decompressed; L4-5 was decompressed similarly. Finally the supra- and interspinous ligaments of both L3-4 and L4-5 were sutured together and each half of the split L-4 spinous process was reapproximated using a strong suture (Fig. 1C).

\section{Outcome Measures}

Clinical and functional outcomes were evaluated us-

TABLE 1. Summary of clinical data of 47 patients with LSS

\begin{tabular}{|c|c|c|c|}
\hline Variable & Modified LSPSL & $\mathrm{CL}$ & p Value \\
\hline \multicolumn{4}{|l|}{ Sex } \\
\hline Male & 18 & 11 & \\
\hline Female & 8 & 10 & \\
\hline Mean age in yrs (range) & $68.3(53-84)$ & $69.4(47-82)$ & 0.65 \\
\hline \multicolumn{4}{|l|}{ Operative levels } \\
\hline 2 & 13 & 10 & \\
\hline 3 & 8 & 9 & \\
\hline 4 & 5 & 2 & \\
\hline \multicolumn{4}{|l|}{ Mean JOA score \pm SD } \\
\hline Preop & $13.0 \pm 4.8$ & $11.9 \pm 2.2$ & 0.300 \\
\hline Postop 12 mos & $23.3 \pm 4.6$ & $23.3 \pm 4.4$ & 0.987 \\
\hline Recovery rate at 12 mos (\%) & $64.2 \pm 27.0$ & $68.7 \pm 25.9$ & 0.619 \\
\hline $\begin{array}{l}\text { Mean recovery rate of subjective symptoms for low-back pain } \\
\text { at } 12 \text { mos postop } \pm \mathrm{SD}(\%)^{*}\end{array}$ & $58.3 \pm 43.2$ & $50.0 \pm 42.9$ & 0.794 \\
\hline Mean surgical duration for single level \pm SD (min) & $25.7 \pm 8.7$ & $22.7 \pm 6.2$ & 0.177 \\
\hline Mean intraop blood loss for single level \pm SD (ml) & $42.4 \pm 37.0$ & $29.5 \pm 32.2$ & 0.213 \\
\hline Mean muscle atrophy rate at 12 mos postop \pm SD (\%) & $7.8 \pm 11.4$ & $22.2 \pm 13.2$ & 0.004 \\
\hline \multicolumn{4}{|l|}{ Bone union rate $(\%)$} \\
\hline Postop 6 mos & 56.3 & & \\
\hline Postop 12 mos & 81.3 & & \\
\hline
\end{tabular}

* The recovery rate of subjective symptoms for low-back pain was assessed using the JOA scoring criteria. 

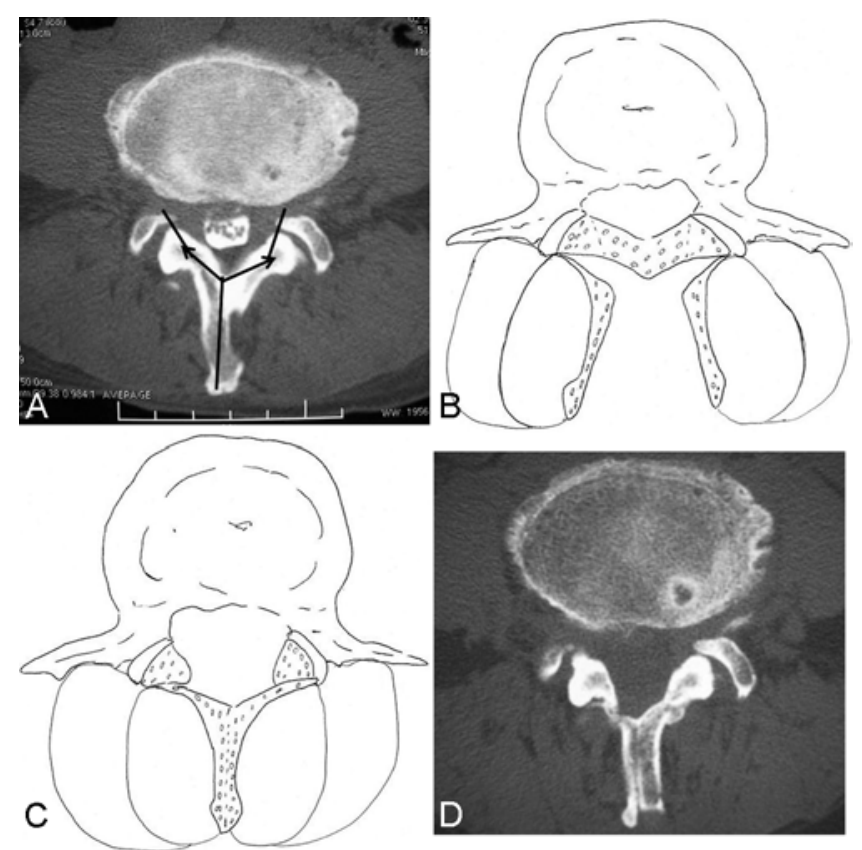

FIG. 1. A: Illustration and axial radiograph depicting the technique for 1-level decompression after spinous process splitting. B: Illustration depicting the divide from the L-4 laminae at an expected angle and retraction of the split spinous process bilaterally with its paravertebral muscles. Copyright Shunsuke Kanbara. Published with permission. C: Illustration showing that each half of the split spinous process is reapproximated using a strong suture. Copyright Shunsuke Kanbara. Published with permission. D: Axial CT scan obtained 12 months after surgery, revealing bone union between the split spinous process and residual laminae.

ing the Japanese Orthopaedic Association (JOA) scale score, ${ }^{7}$ which is composed of 9 points assigned to subjective symptoms, 6 to clinical signs, and 14 to restriction of daily living activities (maximum score $=29$ points). The recovery rate of the JOA score was evaluated using the Hirabayashi method. ${ }^{6}$ Further, to evaluate pre- and postoperative low-back pain, the recovery rate of subjective symptoms for low-back pain was assessed using the JOA scoring criteria. The surgical duration and intraoperative blood loss for each level were analyzed.

\section{Quantitative Analysis of the PVMs}

To evaluate the magnitude of surgical damage to the PVMs, we measured the cross-sectional PVM area on preoperative and 12-month postoperative T2-weighted axial MR images, which were obtained at the lowest operating intervertebral level. Measurement was performed twice on each image, and the mean of the cross-sectional area of PVM was calculated in each patient. The rate of muscle atrophy was calculated using the following formula: atrophy rate $(\%)=(1-$ total postoperative area/total preoperative area) $\times 100 .{ }^{17}$

\section{Bone Union Rates Between the Split Spinous Process and Residual Laminae}

Radiological data for the patients who underwent modified LSPSL were evaluated by axial CT before surgery and 6 and 12 months after surgery at the residual lamina level. However, the patients who underwent modified
LSPSL, but whose bone fusion at 6 and 12 months after surgery was not evaluated with CT, were excluded. If the unilateral or bilateral residual laminae at all decompression levels were fused with the split spinous process, we recognized the bone fusion as successful (Fig. 1D).

\section{Statistical Analysis}

All clinical charts and radiological data of the patients were retrospectively reviewed. The data are presented as means \pm standard deviations. Statistical differences in pre- and postoperative clinical and radiographic parameters after modified LSPSL were compared using the Student $\mathrm{t}$-test. A $\mathrm{p}$ value $<0.05$ was considered statistically significant.

\section{Results \\ Clinical Results}

No major surgery-related complications occurred following modified LSPSL and CL. The mean pre- and 12 -month postoperative JOA scores were $13.0 \pm 4.8$ and $23.3 \pm 4.6$ in modified LSPSL, respectively, and $11.9 \pm 2.2$ and $23.3 \pm 4.4$ in CL, respectively, indicating that the JOA scores were significantly improved after surgery. The mean postoperative JOA scores were not significantly different in either group. The recovery rates at 12 months after surgery were $64.2 \% \pm 27.0 \%$ in modified LSPSL and $68.7 \%$ $\pm 25.9 \%$ in CL. The recovery rates of subjective symptoms for low-back pain at 12 months after surgery were 58.3\% $\pm 43.2 \%$ in modified LSPSL and $50.0 \% \pm 42.9 \%$ in CL. The mean surgical duration and intraoperative blood loss for single-level decompression was 25.7 minutes and 42.4 $\mathrm{ml}$ in modified LSPSL, respectively, and 22.7 minutes and $29.5 \mathrm{ml}$ in CL, respectively. These differences were not statistically significant in both groups (Table 1).

\section{Muscle Invasiveness and Bone Union}

The PVM atrophy rates at 12 months after surgery were $7.8 \% \pm 11.4 \%$ in modified LSPSL and $22.2 \% \pm 13.2 \%$ in CL, respectively (Table 1; Fig. 2). For bone union, 10 $(38.5 \%)$ of the 26 patients who underwent the modified LSPSL procedure were excluded from analysis because of the lack of CT images. The bone union rates between the spinous process and the residual laminae at 6 and 12 months after surgery were $56.3 \%$ and $81.3 \%$, respectively (Table 1).

\section{Discussion}

The LSPSL procedure allows for better exposure of intraspinal nerve tissues, comparable to that achieved by CL, while minimizing damage to posterior supporting structures. We proposed a modified LSPSL procedure, in which laminoplasty is performed instead of laminectomy. JOA scores, the recovery rate of JOA scores and subjective symptoms for low-back pain, and the mean surgical duration and intraoperative blood loss were not significantly different in either procedure. Analysis of our results demonstrated that the muscle atrophy rate was significantly lower in modified LSPSL than in CL. Also, the bone fusion rate at 12 months with modified LSPSL was $81.3 \%$. 

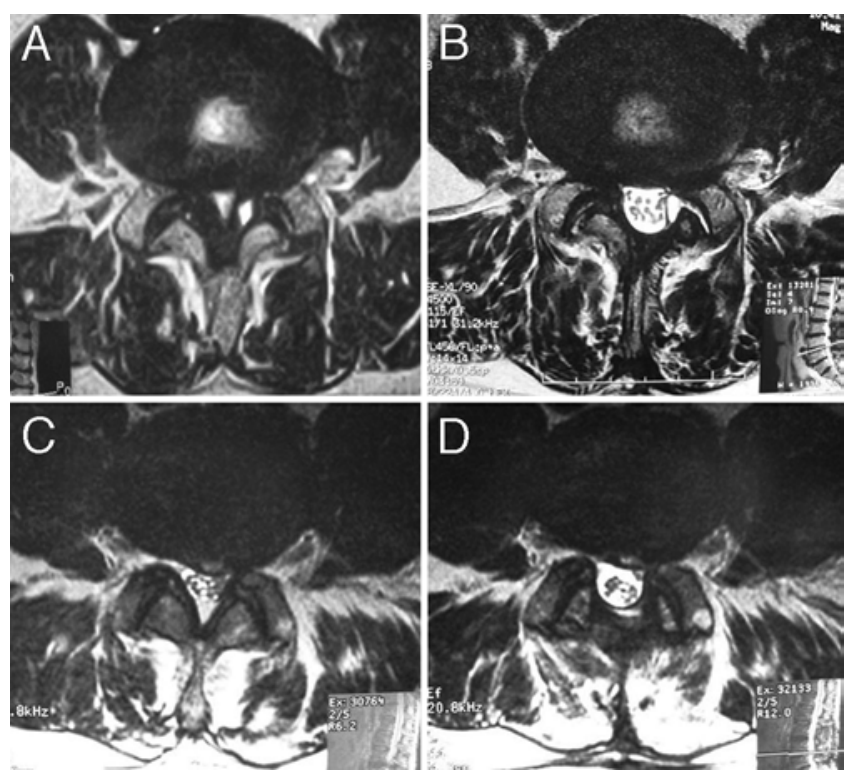

FIG. 2. Preoperative ( $A$ and $C$ ) and 12-month postoperative (B and D) T2-weighted axial MR images from the modified LSPSL ( $A$ and $B$ ) and $\mathrm{CL}(\mathrm{C}$ and $\mathrm{D})$ techniques. These images demonstrated that the PVM atrophy was smaller in modified LSPSL than in CL.

Modified LSPSL can be expected to achieve better longterm spinal stability than conventional spinous processsplitting procedures.

From several anatomical and functional studies of PVM, $4,5,14,15,18$ it is clear that muscle damage can result in postoperative PVM dysfunction. Intraoperative damage to the posterior supporting structures can lead to irreversible PVM atrophy. Kawaguchi et al. ${ }^{12}$ reported that back muscle injury occurred in all patients who underwent posterior lumbar surgery, and these injuries were related to retraction pressure, duration of surgery, and extent of exposure. Moreover, Katz et al. ${ }^{8-11}$ reported that 7-10 years after decompression laminectomy for spinal stenosis, $23 \%$ of patients required revision surgery and $33 \%$ reported severe back or buttock pain. However, several authors have reported many breakthrough procedures. ${ }^{3,16}$ For example, Watanabe et al. ${ }^{17}$ developed the LSPSL procedure as an alternative to lumbar laminectomy. This procedure offers the advantages of a wider surgical working space and optimized visualization, resulting in less muscular damage. Cho et al. ${ }^{2}$ evaluated a minimally invasive spinal surgery technique to correct degenerative LSS involving a splitspinous process laminotomy and discectomy (also known as the Marmot procedure); however, decompression of the lateral recess of the spinal canal by the Marmot procedure is somewhat difficult for a novice neurosurgeon because of the narrow working space. We performed modified LSPSL, a newly developed technique, in which laminoplasty is substituted for laminectomy. Our procedure allows for a wider working space to achieve bone fusion between the split spinous process and residual laminae (Fig. 3 ). In the present study, we used an innovative MRI method to determine the cross-sectional area of PVM after posterior lumbar surgery. This method takes into account both cross-sectional areas on axial MRI. Using a comput-

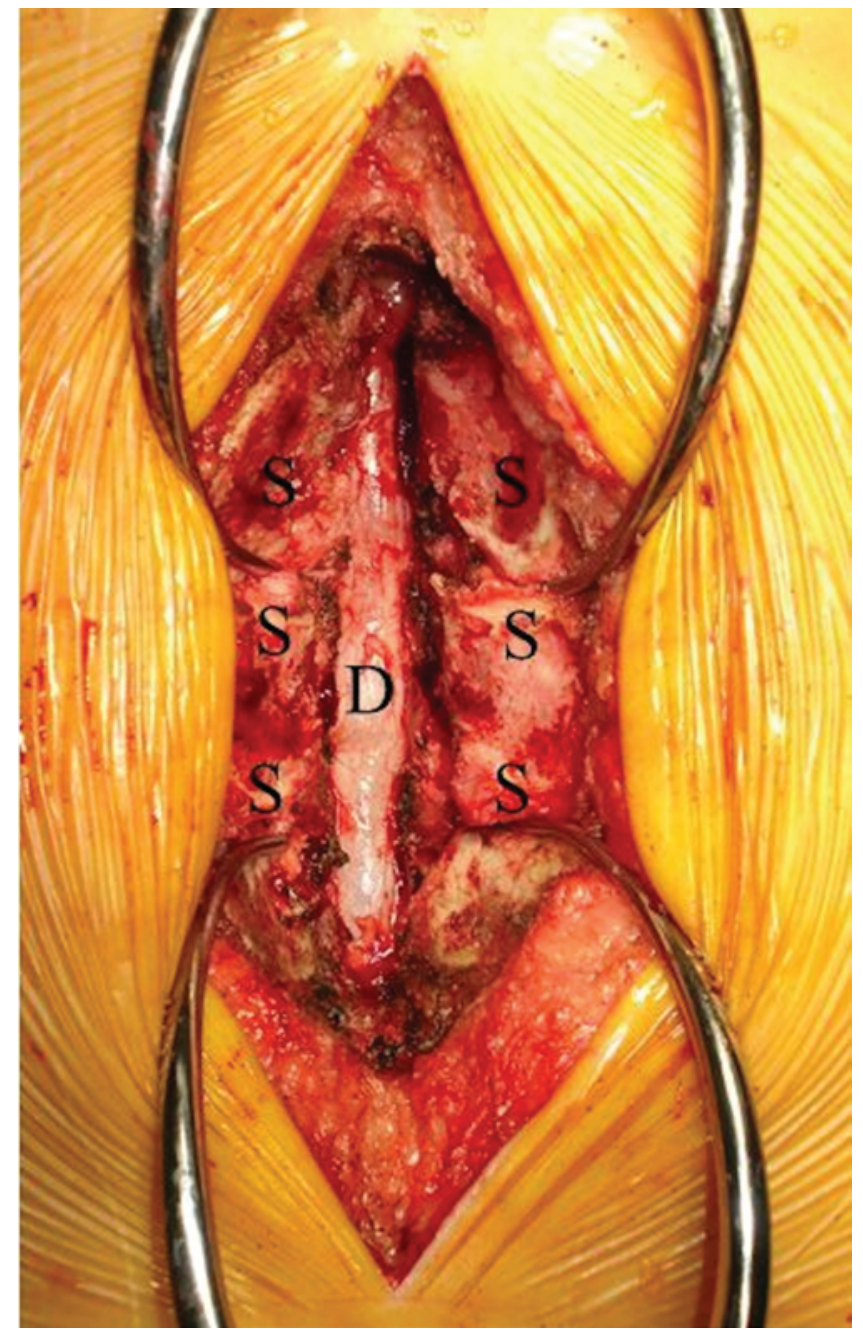

FIG. 3. Intraoperative photograph showing the L-3, L-4, and L-5 split spinous process $(S)$ and the exposed dura (D) after decompression. Figure is available in color online only.

er-based image analysis system, we combined MRI data to measure the cross-sectional area of PVM. Gille et al. ${ }^{5}$ reported that fatty involution of the erector spinae muscles proximal to the decompression level was negligible, although it was more marked distal to the arthrodesis. The vascularization and innervation of these muscles are segmented with numerous vertical anastomoses. Fewer such anastomoses exist in the lower lumbar spine, possibly explaining the relatively severe muscle changes there compared with the sparing of these changes observed in the more proximal muscles. Therefore, we evaluated the intervertebral PVM at the lowest level of surgery because it was expected to receive greater damage than the upper PVMs. The present study showed that the modified LSPSL procedure was less invasive to PVMs than CL, in accordance with the results of past reports. At 12 months after modified LSPSL, the PVM atrophy rate was $7.8 \%$, similar to rates in previous studies ${ }^{13,17}$ (Table 2).

Abumi et al. ${ }^{1}$ reported that the division of the supraspinous and interspinous ligaments did not affect the range of motion. Cho et al. ${ }^{2}$ reported that central splitting and partial 
TABLE 2. Summary of comparative muscle invasive data from 3 studies

\begin{tabular}{lccc}
\hline \multicolumn{1}{c}{ Authors \& Year } & $\begin{array}{c}\text { No. of } \\
\text { Cases }\end{array}$ & $\begin{array}{c}\text { Mean Postop } \\
\text { Muscle Atrophy } \\
\text { Rate } \pm \text { SD (\%) }\end{array}$ & $\begin{array}{c}\text { Postop } \\
\text { Evaluation } \\
\text { (mos) }\end{array}$ \\
\hline Current study & 26 & $7.8 \pm 11.4$ & 12 \\
\hline Watanabe et al., 2005 & 18 & $5.3 \pm 6.6$ & 24 \\
\hline Kojima et al., 2013 & 16 & $13.4 \pm 9.1$ & 12 \\
\hline
\end{tabular}

removal of the interspinous ligaments did not affect spinal stability. The modified LSPSL procedure in the current study not only required the division of the supraspinous and interspinous ligaments but also facilitated bone fusion between the split spinous process and residual laminae. In this study, the bone union rate between the split spinous process and residual laminae was $81.3 \%$. Therefore, the modified LSPSL procedure can be expected to achieve greater spinal stability than conventional splitting procedures. Although the modified LSPSL procedure resulted in limited bone union, postoperative nonfusion of laminae was observed in this study regardless of the number of decompressed levels. Therefore, it is necessary to conduct further studies with a greater number of cases to better evaluate the potential benefits and limitations of modified LSPSL. If bone fusion between the split spinous process and residual laminae is observed, modified LSPSL can be expected to achieve improved long-term spinal stability compared with conventional splitting procedures. The surgical duration of modified LSPSL was a little longer than that of CL because early cases were included. However, the learning curve for the modified LSPSL procedure gradually improved so that in more recent cases the surgical duration was shortened.

This study had some limitations that should be addressed. First, the follow-up periods were relatively short because the intent of this report was to present initial preliminary results; therefore, a longer follow-up period is required to accurately assess long-term spinal stability. Second, bone fusion between the split spinous process and residual laminae could not be examined in all patients.

\section{Conclusions}

The results of the present study revealed good clinical results, in accordance with those of past reports. However, modified LSPSL can be expected to achieve better longterm spinal stability than conventional splitting procedures.

\section{References}

1. Abumi K, Panjabi MM, Kramer KM, Duranceau J, Oxland T, Crisco JJ: Biomechanical evaluation of lumbar spinal stability after graded facetectomies. Spine (Phila Pa 1976) 15:1142-1147, 1990

2. Cho DY, Lin HL, Lee WY, Lee HC: Split-spinous process laminotomy and discectomy for degenerative lumbar spinal stenosis: a preliminary report. J Neurosurg Spine 6:229239, 2007

3. Crock HV, Shiraishi T, Crock MC: Multilevel lumbar canal decompression with preservation of the spinous processes and interspinous ligaments - a review of 34 cases. NeuroOrthopedics 17/18:151-157, 1995
4. Gejo R, Matsui H, Kawaguchi Y, Ishihara H, Tsuji H: Serial changes in trunk muscle performance after posterior lumbar surgery. Spine (Phila Pa 1976) 24:1023-1028, 1999

5. Gille O, Jolivet E, Dousset V, Degrise C, Obeid I, Vital JM, et al: Erector spinae muscle changes on magnetic resonance imaging following lumbar surgery through a posterior approach. Spine (Phila Pa 1976) 32:1236-1241, 2007

6. Hirabayashi K, Watanabe K, Wakano K, Suzuki N, Satomi $\mathrm{K}$, Ishii Y: Expansive open-door laminoplasty for cervical spinal stenotic myelopathy. Spine (Phila Pa 1976) 8:693699, 1983

7. Inoue $\mathrm{S}$, Kataoka $\mathrm{H}$, Tajima T: Assessment of treatment for low back pain. J Jpn Orthop Assoc 60:391-394, 1986

8. Katz JN, Lipson SJ, Chang LC, Levine SA, Fossel AH, Liang $\mathrm{MH}$ : Seven- to 10 -year outcome of decompressive surgery for degenerative lumbar spinal stenosis. Spine (Phila Pa 1976) 21:92-98, 1996

9. Katz JN, Lipson SJ, Larson MG, McInnes JM, Fossel AH, Liang MH: The outcome of decompressive laminectomy for degenerative lumbar stenosis. J Bone Joint Surg Am 73:809-816, 1991

10. Katz JN, Lipson SJ, Lew RA, Grobler LJ, Weinstein JN, Brick GW, et al: Lumbar laminectomy alone or with instrumented or noninstrumented arthrodesis in degenerative lumbar spinal stenosis. Patient selection, costs, and surgical outcomes. Spine (Phila Pa 1976) 22:1123-1131, 1997

11. Katz JN, Stucki G, Lipson SJ, Fossel AH, Grobler LJ, Weinstein JN: Predictors of surgical outcome in degenerative lumbar spinal stenosis. Spine (Phila Pa 1976) 24:2229-2233, 1999

12. Kawaguchi Y, Kanamori M, Ishihara H, Ohmori K, Fujiuchi Y, Matsui H, et al: Clinical symptoms and surgical outcome in lumbar spinal stenosis patients with neuropathic bladder. J Spinal Disord 14:404-410, 2001

13. Kojima M, Seichi A, Inoue H, Endo T, Yamamuro K, Kimura A, et al: [Lumbar spinous process-splitting laminectomy versus conventional laminectomy for lumbar spinal stenosis. a prospective one-year follow-up study.] J Spine Res 4:13931398, 2013 (Jpn)

14. Macintosh JE, Bogduk N: The biomechanics of the lumbar multifidus. Clin Biomech (Bristol, Avon) 1:205-213, 1986

15. Macintosh JE, Valencia F, Bogduk N, Munro RR: The morphology of the human lumbar multifidus. Clin Biomech (Bristol, Avon) 1:196-204, 1986

16. Poletti CE: Central lumbal stenosis caused by ligamentum flavum: unilateral laminotomy for bilateral ligamentectomy: preliminary report of two cases. Neurosurgery 37:343-347, 1995

17. Watanabe K, Hosoya T, Shiraishi T, Matsumoto M, Chiba K, Toyama Y: Lumbar spinous process-splitting laminectomy for lumbar canal stenosis. Technical note. J Neurosurg Spine 3:405-408, 2005

18. Wilke HJ, Wolf S, Claes LE, Arand M, Wiesend A: Stability increase of the lumbar spine with different muscle groups. A biomechanical in vitro study. Spine (Phila Pa 1976) 20:192198,1995

\section{Author Contributions}

Conception and design: Kanbara. Acquisition of data: Kanbara. Analysis and interpretation of data: Kanbara. Critically revising the article: Yukawa, Ito, Machino, Kato.

\section{Correspondence}

Shunsuke Kanbara, Department of Orthopedic Surgery, Chutoen General Medical Center, 1-1 Shobugaike, Kakegawa, Shizuoka 436-8555, Japan. email: shunaly@live.jp. 